\title{
IN THE DECEMBER 2008 ISSUE OF CLINICS
}

\author{
Mauricio Rocha-e-Silva, Editor \\ doi: 10.1590/S1807-59322008000600001
}

This issue of Clinics closes our fourth year of existence and the first in which our impact factor will be formally calculated by the Journal of Citation Reports of ISI Web of Knowledge. During this year we have experienced a $40 \%$ increase in the total of submitted papers, with a $400 \%$ increase in submissions from outside Brazil, to an all time high approximating 60 out of 400 papers. We have also increased the total number of published papers from 91 (in 2007) to 111,10 of which were review articles. We shall be starting the year of 2009 by becoming a monthly journal.

In this issue we publish 18 original research articles and two review articles. We highlight an article by Costa et al, who evaluated the effects of obesity on the pulmonary function, comparing inspiratory and expiratory reserve volumes, as well as maximal voluntary ventilation of 20 obese (BMI $>35 \mathrm{~kg} / \mathrm{m}^{2}$ ) vs. 20 non-obese women. All were sedentary non-smokers aged 20-35. Results clearly show that although obese women have greater inspiratory reserve, they have smaller expiratory reserve and lower maximal voluntary respiration. This indicates damage to the chest mechanics caused by obesity.

Carvalho et al. investigated the relationship between heart rate reserve and oxygen uptake reserve in heart failure patients on optimized and non-optimized beta-blocker therapy. They find that the relationship between the percentage of oxygen consumption reserve and percentage of heart rate reserve is reliable in patients on optimized beta-blocker therapy, but unreliable in non-optimized heart failure patients.

Colombo et al. report the outcomes of laparoscopic partial cystectomy performed in 6 cases at 3 institutions; 3 cases of urachal adenocarcinoma and 3 cases of bladder transitional cell carcinoma and conclude that in selected cases this procedure is feasible and safe, offering a promising and minimally invasive therapeutic alternative.

Conforto et al. investigated changes in excitability of the unaffected hemisphere in chronic stroke patients after

Hospital das Clínicas, Faculdade de Medicina da Universidade de São Paulo - São Paulo/SP, Brazil.

mrsilva36@hcnet.usp.br somatosensory stimulation of the paretic hand. They find that somatosensory stimulation did not influence excitability as measured by motor threshold, short-interval intracortical inhibition or intracortical facilitation. Thy also find that better motor function was correlated with deeper short-interval intracortical inhibition.

Vieira analyzed a Portuguese translation of the postgraduate hospital educational environment measure (PHEEM) questionnaire as a measure of quality of instruction and as a key factor predicting academic achievement, by administering it to 306 medical residents. The conclusion is thatthe teaching section, or quality of instruction is the best indicator of academic achievement.

Moure et al. reviewed the histopathologic diagnoses of 38 cases of primary cicatricial alopecias, sub-typed them into different primary cicatricial alopecias: chronic cutaneous lupus (17), lichen planus pilaris (4), pseudopelade of Brocq (12), folliculitis decalvans (3), dissecting folliculitis (1), and non-specific scarring alopecia (1). They conclude that this approach using systematic criteria allowed for a more accurate diagnosis.

Ciolac et al. applied acute aerobic exercises to 50 hypertensive patients monitored for $24 \mathrm{~h}$ with respect to ambulatory (A) blood pressure after an aerobic exercise session (post-exercise) and a control period (control), in random order. They find that a single bout of aerobic exercise reduced $24 \mathrm{~h}$ ambulatory blood pressure levels in long-term-treated hypertensive patients and increased the percentage of patients reaching normal ambulatory blood pressure values. They suggest that aerobic exercise may have a potential role in blood pressure management of long-term-treated hypertensive.

Kato et al. estimated pretreatment prostate tumor volume based on total serum PSA and define a simple method that allows for estimation of tumor volume before treatment, which can help to establish a better therapeutic strategy for each patient.

Cunha et al. experimentally compared conventional static stretching and muscle chain stretching, as proposed by the global posture reeducation method in patients with chronic 
neck pain. Conventional stretching and muscle chain stretching were equally effective in reducing pain and improving the range of motion and quality of life of 33 female patients with chronic neck pain, both immediately after treatment and after a six-week follow-up period.

Munia et al. compared the results of two levels of videoassisted thoracoscopic sympathectomy (T3-T4 vs. T4) for treating axillary sudoresis at the initial and at six and twelve month follow-ups. They conclude that both techniques were effective for treating axillary hyperhidrosis, but that the $\mathrm{T} 4$ group showed milder compensatory hyperhidrosis and greater patient satisfaction at the one-year follow-up.

Valadares et al. analyzed the prevalence of sexual dysfunction and its associated factors in 315 Brazilian-born women aged 40-65 years, with 11 years or more of formal education, through a cross sectional population based study. They find that sexual dysfunction was positively associated with aging and hot flashes. They also find that having a sexual partner and feeling well were factors associated with lower sexual dysfunction scores.

Martinelli et al. evaluate the quality of life and its association with cardiovascular risk factors in a Community Health Care Program population in a study which included 332 individuals that had attended a specific community event on health care education. They conclude that quality of life was decreased in the presence of cardiovascular risk factors, such as diabetes mellitus, dyslipidemia, and obesity. Conversely, male gender and regular physical activity had protective effects on quality of life.

Andrade-Alegre et al. review open thoracotomy and decortication for chronic empyema as performed in 85 patients and note that these patients were operated with no mortality, $9 \%$ morbidity, no recurrence of empyema and excellent functional results. They suggest that the validation of other surgical approaches should be based on comparative, prospective, and controlled studies.

Branco et al. compare hand-assisted and pure laparoscopic techniques in live donor nephrectomy and observed that the latter technique has some advantages in terms of the warm ischemia time, time to first oral intake, length of hospital stay, and post-operative donor complications.

Galia et al. evaluated the biocompatibility of lyophilized bovine bone manufactured according to a novel preparation process. The following assessments were performed in vitro cytotoxicity, in vivo acute systemic toxicity, in vivo oral irritation potential, in vitro pyrogenic reaction, and bioburden, yelding an end-product with excellent biocompatibility. They conclude that the protocol changes established by them to prepare lyophilized bovine cancellous bone at a semiindustrial scale is reproducible and yielded a product with excellent biocompatibility.

Habib et al. evaluated the effect of ginger extract on the expression of NFKB and TNF- $\alpha$ in liver cancer-induced male Wistar rats and conclude that it significantly reduced the elevated expression of NFKB and TNF- $\alpha$ in rats with liver cancer. They suggest that ginger may act as an anti-cancer and anti-inflammatory agent by inactivating NFKB through the suppression of the pro-inflammatory TNF- $\alpha$.

Potu et al. examined the efficacy of maternally administered petroleum ether extract of the Cissus quadrangularis plant on the development of long bones during the intrauterine development in rats using alizarin red $\mathrm{S}$, alcian blue staining and morphometric analysis by Scion image software, and showed an osteostimulant role of the above plant.

Madhyastha et al. compared vitamin A and leucovorin for the prevention of methotrexate-induced micronuclei production in rat bone marrow through micro nuclei assay of rat bone marrow erythrocytes because it is a predictive assay for chromosomal aberrations, genetic diseases, and carcinogenesis. They conclude that both vitamin A and leucovorin provided significant protection against genetic damage induced by methotrexate and that leucovorin did not differ from vitamin A in its ability to prevent methotrexateinduced micronuclei formation.

Reis and Laranjeira provide an interesting narrative review of the concepts, history, functions, methods, development, and theoretical bases for the use of halfway houses for alcohol dependents, from theoretical bases to the implications for the organization of facilities.

Azoubel et al. provide a review of 30 clinical studies on the use of hyperosmotic-hyperoncotic solutions during surgeries. Reduced positive fluid balance, increased cardiac index, and decreased systemic vascular resistance were the main beneficial effects of using hypertonic solutions.

We also publish three case reports. 